\title{
Roles of $\mathrm{H}^{+}$-ATPase and Proton Motive Force in ATP-Dependent Protein Translocation In Vitro
}

\author{
LINGLING CHEN AND PHANG C. TAI* \\ Department of Metabolic Regulation, Boston Biomedical Research Institute, Boston, Massachusetts 02114, and \\ Department of Microbiology and Molecular Genetics, Harvard Medical School, Boston, Massachusetts 02115
}

Received 14 February 1986/Accepted 8 April 1986

\begin{abstract}
Membrane vesicles from an Escherichia coli mutant with a deletion of the uncBC operon required ATP to translocate proteins, thus ruling out an essential role of $\mathrm{F}_{1} \mathrm{~F}_{0_{0}}-\mathrm{H}^{+}$-ATPase in ATP-dependent protein translocation. Moreover, proteins could be translocated in the absence of proton motive force. At suboptimal ATP concentrations, D-lactate stimulated protein translocation, indicating that proton motive force, although insufficient to support translocation, could facilitate the process.
\end{abstract}

The source of energy for protein translocation across bacterial membranes has been the focus of intensive studies. In intact bacteria, a requirement for an energized membrane was suggested by the observation that dissipation of the proton motive force (PMF) by a proton uncoupler or by valinomycin blocks processing and either insertion or secretion of several membrane and exported proteins $(1,4,5,10$, $11,14)$. Moreover, PMF was found to be involved in protein translocation in vitro in a coupled translation-translocation system (13). However, it is not certain whether the effect of PMF on protein translocation is direct or secondary.

With the finding that in vitro translocation can be accomplished posttranslationally $(2,8)$, it became possible to examine the energy requirements for protein translocation after removal by gel filtration of energy sources used for protein synthesis. (The translocated products were defined as materials [precursor and mature proteins] resistant to pronase digestion and to sedimenting with membrane vesicles.) We have found that translocation of both alkaline phosphatase and OmpA protein into Escherichia coli membrane vesicles requires ATP or an ATP-regenerating system rather than PMF alone (3). The efficiencies of translocation in this system were 10 to 25 and 25 to $35 \%$ for alkaline phosphatase and OmpA protein, respectively $(2,3)$. Moreover, ATP can still support protein translocation, though less efficiently, in the presence of proton uncouplers or with membranes prepared from mutants severely defective in the $\mathrm{F}_{1}$ fraction of the $\mathrm{H}^{+}$-ATPase (3), suggesting that neither PMF nor a functional $\mathrm{H}^{+}$-ATPase is essential for ATPdependent protein translocation. Nevertheless, PMF contributes to the optimal activity since proton uncouplers inhibit the activity by 50 to $70 \%$ (3).

In the earlier experiments involving $F_{1}$-defective membranes, we used an S30 fraction prepared from $E$. coli D10 with normal $\mathrm{H}^{+}$-ATPase, which might have contained trace amounts of the functional $\mathrm{F}_{1}$ portion of the $\mathrm{H}^{+}$-ATPase in solubilized form capable of complementing the $\mathrm{F}_{0}$ portion of the $\mathrm{F}_{1}$-defective membranes to provide ATP-dependent protein translocation. To eliminate this possibility and to determine whether the $\mathrm{F}_{0}$ portion of $\mathrm{H}^{+}$-ATPase might be involved in translocation (e.g., by providing a channel or pore), experiments were carried out with mutant CK1801

\footnotetext{
* Corresponding author.
}

$\left(\mathrm{F}^{-} \Delta\right.$ lacU169 araD139 thiA rpsL relA $\left.\Delta u n c B C\right)$, a derivative of MC4100 (obtained from C. Kumamoto of Stanford University) whose genes for both $\mathrm{F}_{1}$ and $\mathrm{F}_{0}$ of $\mathrm{H}^{+}$-ATPase are entirely deleted. As expected, this mutant could not grow or revert to growth with succinate as the carbon source. Membrane vesicles from this strain CK1801 were prepared and used for translocating alkaline phosphatase and OmpA protein in the translocation assay described previously, except that membranes were suspended in $10 \mathrm{mM}$ Tris hydrochloride ( $\mathrm{pH} 7.6)-50 \mathrm{mM} \mathrm{KCl}$ without $\mathrm{Mg}^{2+}$ (3). Membrane potential was measured indirectly as the absorbance changes of the potential-sensitive dye oxonol VI (12) at a dual wavelength of 594 to $630 \mathrm{~nm}$ in a double-beam dualwavelength spectrophometer (model 557; The Perkin-Elmer Corp.). Membrane vesicles containing $80 \mu \mathrm{g}$ of protein were used. CK1801 membranes could not generate a membrane potential with ATP- $\mathrm{Mg}^{2+}$, while $\mathrm{H}^{+}$-ATPase containing membranes of strain D10 generated 3.0 units (absorbance times 100 ) of membrane potential. However, with D-lactate the CK1801 membranes generated a membrane potential of 6.6 which was much higher than the 0.8 membrane potential generated by the D10 membranes.

Strain CK1801 membrane vesicles, devoid of $\mathrm{F}_{1} \mathrm{~F}_{0}-\mathrm{H}^{+}$. ATPase, could use ATP for protein translocation in the presence of $5 \mathrm{mM} \mathrm{Mg}{ }^{2+}$ almost as efficiently as, and in some cases more efficiently than, D10 membranes with functional $\mathrm{H}^{+}$-ATPase (Table 1; Fig. 1, lanes $b$ and $\mathrm{f}$ ) but could not of course use D-lactate for translocation (Table 1). (CK1801 membranes used in the experiment were somewhat more active than D10 membranes in translocation, presumably because of variation in preparations or strain differences.) These data thus ruled out any essential involvement of $\mathrm{F}_{1} \mathrm{~F}_{0^{-}}$ $\mathrm{H}^{+}$-ATPase in ATP-dependent translocation. The apparent indispensable requirement described earlier (9) for the $F_{1}$ portion of $\mathrm{H}^{+}$-ATPase for protein translocation in a coupled transcription-translation system probably reflected a difference in the system used or a secondary effect of $\mathrm{H}^{+}$-ATPase, presumably through the generation of PMF at least in part to replenish ATP in that system $(3,13)$ and also to reduce the amount of ATP required for translocation (see below).

Another observation supporting the notion that a functional $\mathrm{H}^{+}$-ATPase requiring $\mathrm{Mg}$-ATP for activity is not directly involved in ATP-dependent translocation was that both $\mathrm{H}^{+}$-ATPase-containing membranes from strain D10 and 
TABLE 1. Effects of $\mathrm{H}^{+}$-ATPase, FCCP, and $\mathrm{Mg}^{2+}$ on ATP-dependent translocation ${ }^{a}$

\begin{tabular}{|c|c|c|c|c|c|c|}
\hline \multirow{3}{*}{ Addition } & \multicolumn{6}{|c|}{ \% Translocation activity in strain: } \\
\hline & \multicolumn{3}{|c|}{ CK1801 ( $\triangle$ ATPase) } & \multicolumn{3}{|c|}{ D10 (ATPase $^{+}$) } \\
\hline & APase & pOmpA & OmpA & APase & pOmpA & OmpA \\
\hline ATP & $100(156)^{b}$ & $100(208)^{b}$ & $100(181)^{b}$ & 100 & 100 & 100 \\
\hline $\mathbf{A T P}+\mathbf{F C C P}$ & 90 & 73 & 95 & 90 & 86 & 92 \\
\hline $\mathrm{ATP}+\mathbf{M g}^{2+}$ & 98 & 115 & 100 & 97 & 100 & 118 \\
\hline $\mathrm{ATP}+\mathrm{Mg}^{2+}+\mathrm{FCCP}$ & & & & 21 & 42 & 40 \\
\hline D-Lactate & $\mathbf{0}$ & 0 & $\mathbf{0}$ & $\mathbf{0}$ & $\mathbf{0}$ & 0 \\
\hline D-Lactate $+\mathbf{M g}^{2+}$ & $\mathbf{0}$ & $\mathbf{0}$ & $\mathbf{0}$ & 10 & 25 & 30 \\
\hline
\end{tabular}

${ }^{a}$ Translocation was carried out as described previously (3), with $3 \mathrm{mM}$ ATP-Tris, $5 \mu \mathrm{M}$ FCCP, $5 \mathrm{mM}$ magnesium acetate, and $10 \mathrm{mM}$ D-lactate where indicated. The fluorograms of the translocational products were scanned, and the data were expressed as percent translocation activity, $\mathrm{ATP}$ without added $\mathrm{Mg}^{2+}$ being $100 \%$, that of alkaline phosphatase (APase), precursor of OmpA protein (pOmpA), and OmpA protein.

$b$ Number in parentheses is the percent activity compared with that in D10 membrane vesicles.

$\mathrm{H}^{+}$-ATPase-depleted membranes from strain CK1801 used ATP to translocate proteins whether $5 \mathrm{mM} \mathrm{Mg}^{2+}$ was present or not (Fig. 1, lanes b, c, f, and g). (It should be noted, however, that residual $\mathbf{M g}^{2+}$ bound to various compounds might have been present in the system.) Without added $\mathrm{Mg}^{2+}, 5 \mu \mathrm{M}$ carboxylcyanide- $p$-trifluoromethoxyphenylhydrazone (FCCP), which collapses PMF and completely inhibits D-lactate-dependent translocation (3), had little or no effect on ATP-dependent translocation in both kinds of membranes (Table 1; Fig. 1, lanes $d$ and $h$ ), but in the presence of added $\mathbf{M g}^{2+}$, FCCP inhibited translocation with $\mathrm{H}^{+}$-ATPase-containing membranes by 60 to $80 \%(3$; Table 1). Similarly, valinomycin (plus $\mathrm{K}^{+}$) or the $\mathrm{H}^{+}$ATPase inhibitor $N, N^{\prime}$-dicyclohexylcarbodiimide had little effect on ATP-dependent translocation in the absence of added $\mathrm{Mg}^{2+}$. These results provided additional evidence that in the absence of functional $\mathrm{H}^{+}$-ATPase PMF has no significant role in ATP-dependent translocation. The results are

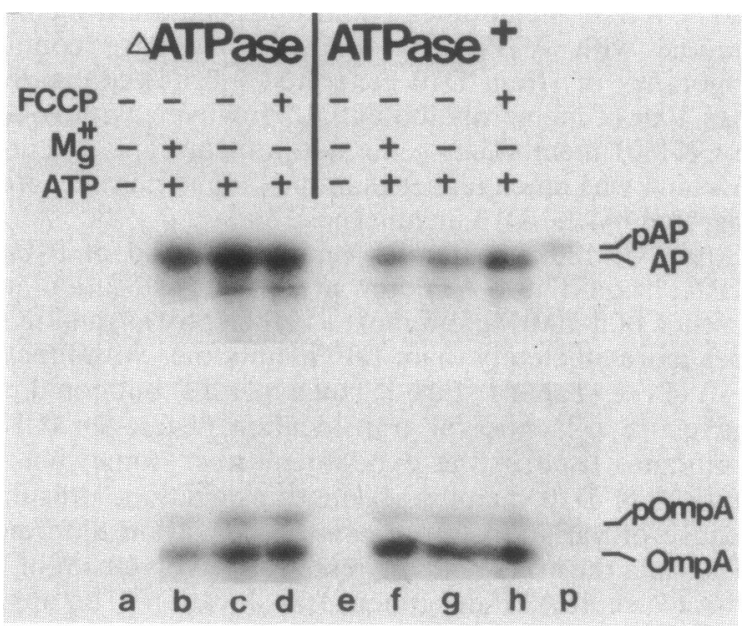

FIG. 1. Effects of $\mathrm{H}^{+}$-ATPase, FCCP, and $\mathrm{Mg}^{2+}$ on ATPdependent translocation. Translocation into membrane vesicles was carried out, and the translocated products were detected by polyacrylamide gel electrophoresis essentially as described previously (3), except as noted below. Lanes: a to d, strain CK1801 membranes; e to h, strain D10 membranes; $a$ and e, no ATP; b and f, 3 $\mathrm{mM}$ ATP plus $5 \mathrm{mM}$ magnesium acetate; $\mathrm{c}$ and $\mathrm{g}, 3 \mathrm{mM}$ ATP; $d$ and $\mathrm{h}, 3 \mathrm{mM}$ ATP and $5 \mu \mathrm{M}$ FCCP. Precursor markers (translational products) in lane $p$ are as follows: pAP, precursor of alkaline phosphatase; pOmpA, precursor of outer membrane protein OmpA. The upper and lower parts of the fluorograms were obtained after 5 and $16 \mathrm{~h}$ of exposure, respectively, to Kodak X-5 film. consistent with the observation that $E$. coli cells can grow on a medium containing a proton uncoupler, such as FCCP (7), implying that PMF is not obligatory for cell growth and related essential processes, such as protein translocation. On the other hand, under certain conditions, e.g., with functional $\mathrm{H}^{+}$-ATPase, PMF contributed to optimal protein translocation.

The role of PMF in ATP-dependent protein translocation was further studied at various concentrations of ATP in the absence of added $\mathrm{Mg}^{2+}$. Translocation with wild-type strain D10 membranes was dependent on ATP, reaching maximal activity at $3 \mathrm{mM}$ (Table 2). (The optimal concentration of ATP for translocation varied from 3 to $5 \mathrm{mM}$ with different membrane preparations.) The addition of $D$-lactate, which generates PMF through oxidative respiration, had no significant effect on the kinetics and the amount of translocation at 3 or 5 mM ATP (Table 2). Similar results were obtained with

TABLE 2. Effects of D-lactate and NADH on ATP-dependent translocation ${ }^{a}$

\begin{tabular}{|c|c|c|c|}
\hline \multirow{2}{*}{ Added concentration ( $\mathrm{mM}$ ) } & \multicolumn{3}{|c|}{$\%$ Translocation activity ${ }^{b}$ of: } \\
\hline & APase & pOmpA & OmpA \\
\hline \multicolumn{4}{|l|}{ ATP (mM) } \\
\hline 0.5 & 6 & 25 & 15 \\
\hline 1 & 34 & 57 & 69 \\
\hline 3 & 100 & 100 & 100 \\
\hline 5 & 94 & 90 & 92 \\
\hline \multicolumn{4}{|l|}{ D-Lactate + ATP } \\
\hline 0.5 & 15 & 48 & 46 \\
\hline 1 & 75 & 83 & 83 \\
\hline 3 & 75 & 97 & 92 \\
\hline 5 & 70 & 90 & 80 \\
\hline \multicolumn{4}{|l|}{ NADH + ATP } \\
\hline 3 & 77 & 85 & 78 \\
\hline 5 & 93 & 85 & 94 \\
\hline \multicolumn{4}{|l|}{$\mathrm{FCCP}+\mathrm{D}$-lactate $+\mathrm{ATP}$} \\
\hline 0.5 & $9(60)$ & $22(46)$ & $23(50)$ \\
\hline 1 & $19(25)$ & $54(65)$ & $38(45)$ \\
\hline 3 & $53(71)$ & $68(70)$ & $65(70)$ \\
\hline 5 & $50(71)$ & $55(61)$ & $51(63)$ \\
\hline
\end{tabular}

${ }^{a}$ Translocation was carried out with strain D10 membranes in the absence of added $\mathrm{Mg}^{2+}$ and with ATP at the concentrations indicated, and the data were analyzed as described in the footnote to Table 1. D-Lactate, NADH, and FCCP concentrations were $10 \mathrm{mM}, 3 \mathrm{mM}$, and $5 \mu \mathrm{M}$, respectively. Translocation activity for each APase, pOmpA, and OmpA at $3 \mathrm{mM}$ ATP was taken as $100 \%$.

${ }^{b}$ Numbers in parentheses are percents activity without FCCP. 
TABLE 3. Effects of D-lactate on ATP-dependent translocation with strain CK1801 membranes devoid of $\mathrm{H}^{+}$-ATPase ${ }^{a}$

\begin{tabular}{lccc}
\hline \multirow{2}{*}{ Added concentration (mM) } & \multicolumn{3}{c}{$\%$ Translocation activity } \\
\cline { 2 - 4 } & APase & pOmpA & OmpA \\
\hline ATP & 26 & 33 & 59 \\
1 & 100 & 100 & 100 \\
3 & 76 & 87 & 95 \\
5 & & & \\
& 132 & 120 & 112 \\
D-Lactate + ATP & 246 & 142 & 124 \\
1 & 145 & 123 & 113 \\
3 & & & \\
5 & 121 & 115 & 130 \\
& 115 & 104 & 121 \\
NADH + ATP & & & \\
3 & & & \\
5 & $13(10)$ & $24(20)$ & $32(29)$ \\
& $62(25)$ & $61(43)$ & $80(64)$ \\
D-Lactate + FCCP + ATP & $57(39)$ & $53(43)$ & $63(56)$ \\
1 & &
\end{tabular}

${ }^{a}$ Translocation was carried out with strain CK1801 membranes deleted of $\mathrm{H}^{+}$-ATPase, and activity was expressed as described in the footnote to Table 2.

${ }^{b}$ Numbers in parentheses are percents activity without FCCP.

NADH, another compound that generates PMF through respiration (Table 2). However, FCCP decreased translocation in the presence of D-lactate (therefore PMF) plus 3 or 5 mM ATP to a level lower than that observed at 3 or $5 \mathrm{mM}$ ATP alone, even though D-lactate did not stimulate the translocation (Table 2). At lower ATP concentrations $(0.5$ and $1 \mathrm{mM}$ ), which supported suboptimal activity, D-lactate increased translocation to a near optimal level in an FCCP. sensitive manner (Table 2). Under these conditions, when PMF was provided through D-lactate, the minimal concentration of ATP required for optimal translocation activity was between 0.5 and $1 \mathrm{mM}$.

Similar experiments were carried out with $\mathrm{H}^{+}$-ATPasedevoid strain CK1801 membrane, which, in addition to the lack of $\mathrm{H}^{+}$-ATPase, probably had a different membrane protein composition, e.g., enzymes of the respiratory chain, than strain D10 wild-type membranes (6). The optimal ATP concentration for translocation was $3 \mathrm{mM}$, and D-lactate or $\mathrm{NADH}$ increased translocation to various degrees at all concentrations tested (Table 3 ). It should be noted that with CK1801, but not with D10 membranes, the addition of D-lactate or NADH consistently stimulated translocation even at optimal ATP, perhaps due to a difference in membrane topology and composition or because of a higher level of membrane potential through respiration with the $\mathrm{H}^{+}$ATPase-deleted membranes. As with the D10 membrane, FCCP reduced the stimulation of translocation by D-lactate (Table 3). These results further substantiated our earlier suggestion (3) that, although PMF is neither essential nor sufficient for protein translocation, it contributes to optimal activity, and as shown here, this contribution was more pronounced at suboptimal concentrations of ATP. Moreover, it appeared that, although proteins could be translocated efficiently with ATP and no PMF, FCCP greatly inhibited protein translocation in the presence of PMF, even with excess ATP (Tables 2 and 3). These observations may explain the in vivo findings $(1,4,5,11,14)$ that protein export is defective in cells treated with proton uncouplers, even with normal ATP levels (1); i.e., protein translocation is impaired with the collapse of proton gradient, resulting in the slowdown and jam-up of the translocation pathway which eventually leads to subsequent accumulation of the precursor.

The exact functions of ATP and its hydrolysis in protein translocation (3) are not known. It appears that some, but not all, of the functions can be fulfilled by PMF, since maximal translocation activity was observed either with optimal ATP concentrations or with suboptimal ATP concentrations plus compounds that generated PMF. We suggest that the function of ATP includes at least two yet unknown components; one of them is essential for protein translocation, and the other merely facilitates the process and can be replaced by PMF. The two components differ in their dependence on ATP concentration, the essential component being saturated at low ATP $(0.5$ to $1.0 \mathrm{mM})$ and the facilitating component requiring higher ATP concentrations ( 3 to $5 \mathrm{mM}$ ). One possible basis for the facilitating activity is the maintenance of the overall membrane topology and proper protein conformation involved in protein translocation, such as interaction of the precursor and signal peptidase. This step may be achieved either by FCCPinsensitive ATP hydrolysis not related to $\mathrm{H}^{+}$-ATPase or perhaps more effectively by FCCP-sensitive PMF, including but not restricted to the component generated by $\mathrm{H}^{+}$. ATPase.

We thank J. S. Hong for stimulating discussions, M. Pringle for determining membrane potential, B. D. Davis and H. Paulus for helpful comments, C. Kumamoto for providing the strain, and D. Syrigos for typing the manuscript.

This work was supported in part by Public Health Service grant GM34766 and Biomedical Research Support grant RR05711 from the National Institutes of Health and by the Harvard Milton Fund.

\section{LITERATURE CITED}

1. Bakker, E. P., and L. L. Randall. 1984. The requirement for energy during export of $\beta$-lactamase in Escherichia coli is fulfilled by the total protonmotive force. EMBO J. 3:895-900.

2. Chen, L. L., D. Rhoads, and P. C. Tai. 1985. Alkaline phosphatase and OmpA protein can be translocated posttranslationally into membrane vesicles of Escherichia coli. J. Bacteriol. 161:973-980.

3. Chen, L. L., and P. C. Tai. 1985. ATP is essential for protein translocation into Escherichia coli membrane vesicles. Proc. Natl. Acad. Sci. USA 82:4384-4388.

4. Daniels, C. J., D. G. Bole, S. C. Quay, and D. L. Oxender. 1981. A role for membrane potential in the secretion of protein into the periplasm of Escherichia coli. Proc. Natl. Acad. Sci. USA 77:4669-4673.

5. Enequist, H. G., T. R. Hirst, S. J. S. Hardy, S. Harayama, and L. L. Randall. 1981. Energy is required for maturation of exported proteins in Escherichia coli. Eur. J. Biochem. 116:227-233.

6. Haddock, B. A., and C. W. Jones. 1977. Bacterial respiration. Bacteriol. Rev. 41:47-99.

7. Kinoshita, N., T. Unemoto, and H. Kobayashi. 1984. Proton motive force is not obligatory for growth of Escherichia coli. J. Bacteriol. 160:1074-1077.

8. Muller, M., and G. Blobel. 1984. In vitro translocation of bacterial proteins across the plasma membrane of Escherichia coli. Proc. Natl. Acad. Sci. USA 81:7421-7425.

9. Muller, M., and G. Blobel. 1984. Protein export in Escherichia coli requires a soluble activity. Proc. Natl. Acad. Sci. USA 81:7737-7741.

10. Murén, E. M., and L. L. Randall. 1985. Export of $\alpha$-amylase by Bacillus amyloliquefaciens requires proton motive force. J. Bacteriol. 164:712-716.

11. Pages, J. M., and C. Lazdunski. 1982. Maturation of exported proteins in Escherichia coli. Requirement of energy, site and 
kinetics of processing. Eur. J. Biochem. 124:561-566.

12. Pringle, M. J., and D. R. Sanadi. 1984. Effect of $\mathrm{Cd}^{2+}$ on ATP-driven membrane potential in beef heart mitochondrial $\mathrm{H}^{+}$-ATPase: a study using the voltage-sensitive probe oxonol VI. Membr. Biochem. 5:225-241.

13. Rhoads, D. B., P. C. Tai, and B. D. Davis. 1984. Energy- requiring translocation of the OmpA protein and alkaline phosphatase of Escherichia coli into inner membrane vesicles. J. Bacteriol. 159:63-70.

14. Zimmermann, R., and W. Wickner. 1983. Energy and intermediates of the assembly of protein OmpA into the outer membrane of Escherichia coli. J. Biol. Chem. 258:3920-3925. 